\title{
Methods Related to Herbicide Dissipation or Degradation under Field or Laboratory Conditions
}

\author{
Thomas C. Mueller and Scott A. Senseman*
}

Key words: Degradation, herbicide, LCMS, soils.

Herbicide degradation in soil is a major research issue, as evidenced by the number of refereed articles on the subject (Figure 1). The approximate number of total citations per year has increased from about 70 in the mid-1990s to approximately 170 per year in the last few years (Figure 1a). From a weed science perspective, publications in the journals Weed Science and Weed Technology have tended to decline from an average of eight per year in the 1995 to 2005 interval to about three per year in the last $10 \mathrm{yr}$ (Figure 1b). This discrepancy of total citations vs. Weed Science Society of America journals may be due to funding availability and other more immediate research needs in weed science. Other reasons might be a lack of reader interest (indicating low potential impact in this specific topic area) or the perception that Weed Science and Weed Technology are light venues for such papers and therefore not the first choice for publication. The authors believe this research topic to be important and relevant to the discipline of weed science, even more so as herbicide use patterns become more complicated because of glyphosate-resistant (GR) weeds.

Several review articles discuss soils and herbicide behavior (Locke and Bryson 1997; Sarmahm and Sabadie 2002), and several reviews or books contain chapters on methodologies for assessing herbicide behavior or fate in the field. Examples include: Agrochemical Environmental Fate: State of the Art (Leng et al. 1995), Pesticide Environmental Fate: Bridging the Gap between Laboratory and Field Studies (Phelps et al. 2002), and Terrestrial Field Dissipation Studies: Purpose, Design, and Interpretation (Arthur et al. 2003). Another excellent comprehensive reference is the North American Free Trade Agreement guidance document for conducting terrestrial field dissipation studies (U.S. EPA 2006).

\footnotetext{
DOI: 10.1614/WS-D-13-00157.1

* Professors, Department of Plant Sciences, University of Tennessee, Knoxville, TN 37996. Corresponding author's Email: tmueller@utk.edu

No abstract on this paper due to inclusion in special edition of methods paper.
}

Many soil studies of herbicide behavior are conducted to support registration with the U.S. Environmental Protection Agency (U.S. EPA; Corbin et al. 2006). Those studies, consisting of field and laboratory scenarios followed by chemical analysis, are conducted under good laboratory procedures (GLPs). The conductance of these studies is directed and guided under very specific and rigorous conditions. Although many of the concepts and ideas discussed in this paper are relevant to these studies, many are more germane to the non-GLP scientist. Discussion of laboratory herbicide degradation will be included; however, the focus of the paper will be herbicide behavior in a field environment.

To begin, there are a few terms to define and discuss. With respect to the residence time a herbicide remains in a given portion of the soil matrix, several related terms include degradation, dissipation, and persistence. Degradation is defined as a substantive change in the molecular makeup of the given herbicide, with a component of the parent molecule removed by some process to form a metabolite or metabolites. Dissipation is considered to be the sum of all possible outcomes of the parent herbicide. For example, the parent herbicide could be volatilized and moved off-site which would reduce the herbicide concentration. A herbicide could dissipate by being leached into subsurface zones below the sampling area or removed from the field in surface runoff. Neither of these transformation processes necessarily alters the chemical form of the herbicide. A herbicide molecule can dissipate by the process of chemical or microbial degradation which, as previously defined, indicates a chemical change in the parent. The inverse process of dissipation is herbicide persistence. Persistence is often considered to be a negative connotation, such as herbicide persistence that damages rotational crops or herbicide persistence that causes contamination of rivers and groundwater aquifers. On the other hand, herbicide persistence can allow for residual weed control to provide maximum agricultural productivity in managed ecosystems. 

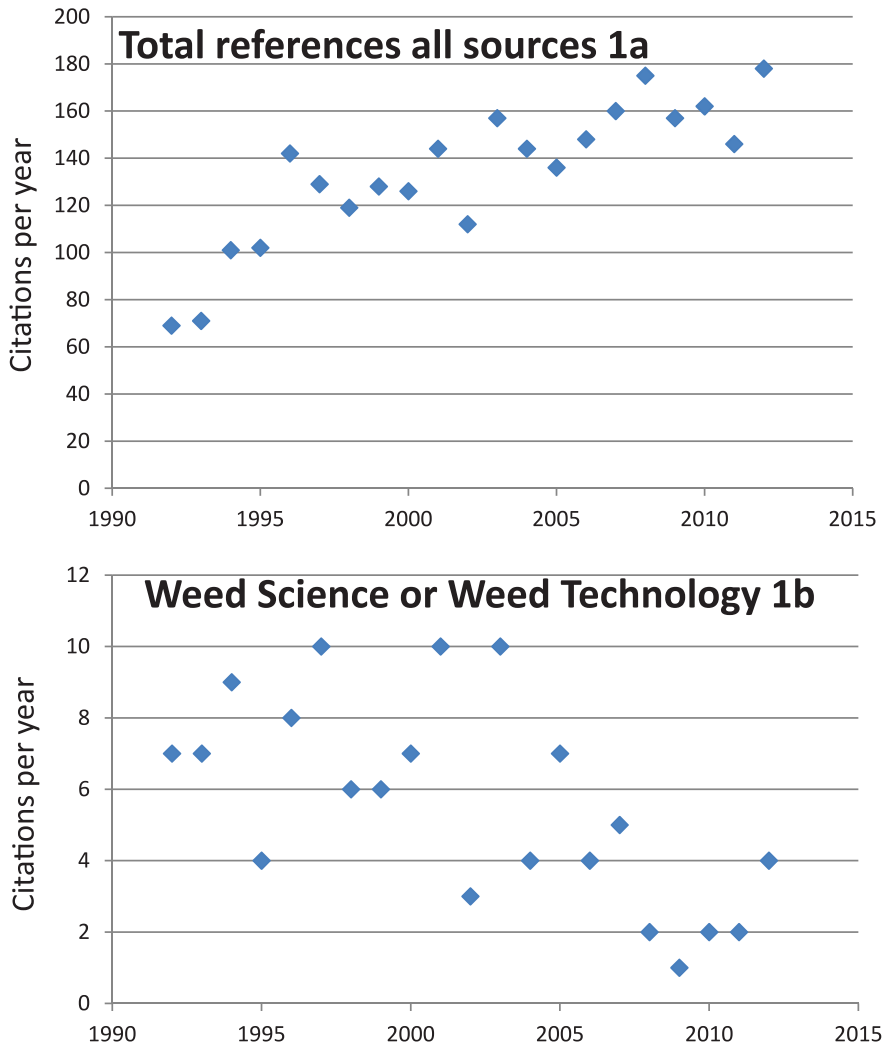

Figure 1. Number of refereed journal articles found in Web of Science using the search terms "Herbicide" and "degradation" and "soil" for each year from 1992 to 2012. Top portion (a) of figure represents all searchable citations, lower portion (b) represents searchable citations from a Weed Science and/or Weed Technology citation.

The principles described in this paper apply to a broad range of experiments examining herbicide fate. For ease of discussion, we will describe the PRE herbicide application to a field into which an agronomic crop has just been planted. The authors realize that many of these studies are conducted with no crop present, but the presence of a crop has the potential to affect the results (Gallaher and Mueller 1996). The general sections to discuss the methods are divided into the following:

- Site selection

- Experimental design

- Sample collection

- Sample storage and processing

- Chemical concentration determination

- Regression analysis

\section{Protocols and Methods}

Site Selection. An important consideration in the successful conductance of any type of field study is site selection. Important considerations when choosing an area include the lack of any detectable residues of the component of interest, which would confound the results upon analysis. The area should represent the actual environment in which the herbicide will be used, including soil characteristics, tillage system, crops, fertility, irrigation, and so forth. A challenge to conducting these types of studies in no-tillage systems is the presence of plant residue from previous years, which can complicate the soil sample collection process. Some sampling equipment may not penetrate heavy plant residue. The presence of plant material within the collected sample also may make matrix homogenization more difficult. The full effect of plant residue on the soil sampling process is unclear. If one removes the plant residue before soil sampling, one is assuming the herbicide is washed off the residue into the soil. Important considerations for soil sample collection have been discussed previously (Blumhorst and Mueller 1997).

It is usually preferable to conduct a field study under weed-free conditions. This avoids confounding effects due to the presence of more or fewer weeds in a given plot based on what herbicide is present (Gallaher and Mueller 1996); also, reduced vegetation can allow for easier sample collection. Maintaining studies in a weed-free environment without detectable herbicide residues is much easier with the advent of herbicide-resistant crops such as GR or glufosinate-resistant varieties. Other aspects of site selection include ease of access to the site, knowledge of prior crop history, distance to weather station, long-term security of the site (for multiyear studies), and availability of the appropriate equipment to establish and maintain the crops.

Experimental Design. Experimental design is important to herbicide dissipation in soil studies. The number of treatments is usually small, sometimes only two (e.g., a treated and a nontreated plot). It is essential always to include a nontreated control plot for comparison purposes, so that if chemical analysis shows interfering components, they can be explained as a nonherbicidal factor. Selecting a location with no prior use of the examined herbicide should prevent residues from previous applications. A good practice, however, is to conduct analysis before herbicide application to verify the lack of these residues or their metabolites. Some studies, such as those investigating enhanced degradation due to previous use, by definition must have a use history of the chemical in previous years. 


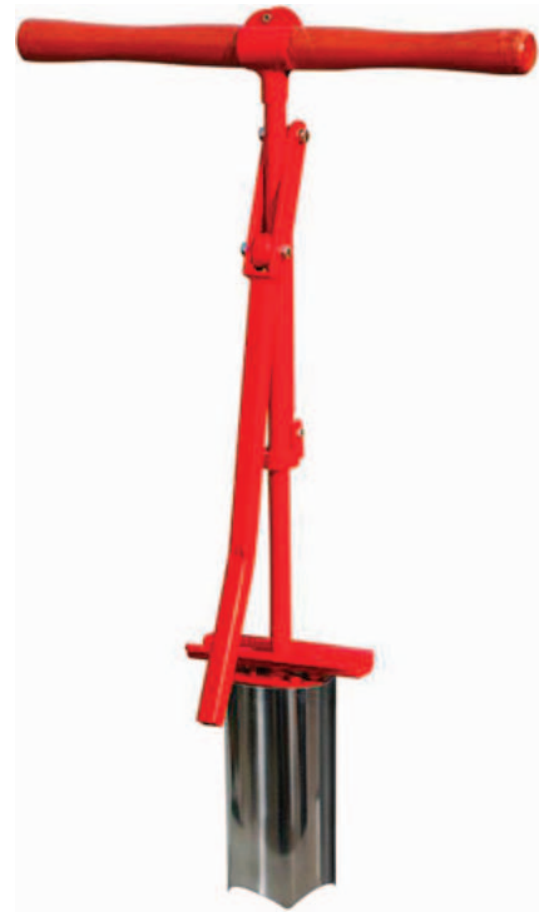

Figure 2. Golf cup cutter can be used to collect surface soil samples.

The next important consideration in the experimental design is the sample collection interval. Most herbicide dissipation curves are not linear and are usually first-order or biphasic in their shape (Aldworth and Jackson 2008; Brown et al. 1996). Given this observation (nonlinear decline in herbicide concentration soon after application), it is appropriate to have a more intensive sampling interval closer to the time of herbicide application, such as $0,1,2,4,8,16$, and $32 \mathrm{~d}$ after treatment (DAT) for a herbicide with a half-life expected to be less than $7 \mathrm{~d}$. A possible sampling interval for a herbicide with an estimated half-life of $50 \mathrm{~d}$ might be $0,7,14,21,28,40,50,60,80,100$, and 120 DAT. The study duration should be sufficient to allow for the majority of the applied compound to dissipate. An important reminder is that the most critical single sampling event of the entire study is 0 DAT, or immediately after application. The entire regression analysis is based upon chemical quantity loaded into the soil. Important considerations of these 0 DAT samples have been previously discussed (Blumhorst and Mueller 1997).

There is always a trade-off between having a large number of time point estimates of herbicide concentration and a small enough number of experimental units that the researcher can afford to collect, process, extract, and analyze on the basis of time and funding constraints. One aspect to consider is that a given target sampling time is just an estimate, so if one deviates from the target sampling time, it is really not a major experimental error since regression analysis can account for the altered data point in time.

Sample Collection. Sample collection is dependent on the data requirements of the study. In most normal agronomic scenarios, the greatest herbicide concentrations are found near the soil surface. This soil region is also the primary area responsible for biological effects such as weed control or carryover into rotational crops. A possible equipment choice to collect samples from the 0 to $10 \mathrm{~cm}$ soil depth is to use a golf cup cutter (Figure 2). This equipment is inexpensive, readily available at golf supply outlets, robust and rugged, and designed to cut through soil and residue, and it collects a fairly large and representative soil sample. Another advantage of using a golf cup cutter is that the handle is designed to expel the soil sample after collection, thus the sample can be placed directly into heavyduty plastic bags for storage before analysis. Special circumstances may require altered sampling methods, such as moist soil high in clay content or when rocks are present, both of which may prevent soil sampler insertion into the soil profile. Alternatively, samples of very coarse soils (sands) can be difficult to collect when it is dry because the soil may have no structure and is not captured by the sampler. Field sites with less surface plant residue allow for easier soil sample collection. Once the sample has been collected, it is imperative to place the labeled and sealed soil sample bag directly into a cooler to prevent photo, microbial, or chemical degradation. It is preferable to have a freezer in close physical proximity to the field site to store the samples immediately. A cautionary note is that plastic bags can often contain phthalate esters or plasticizers that can interfere with later chemical analysis.

The general purpose of the study normally dictates the depth of soil sample collection. To support registration, samples must be collected from various depths throughout the soil profile to a depth sufficient to encompass the vertical distribution of the pesticide and its major transformation products at each sampling time (Corbin et al. 2006). This typically is to a depth of $1 \mathrm{~m}$, with the profile divided into six or more depths at approximately $15-\mathrm{cm}$ intervals. If samples from subsurface soil zones are required, care should be taken to avoid contamination from the surface $(\sim 0$ to $8 \mathrm{~cm})$ to subsurface soil samples. Since the herbicide concen- 
tration is usually greatest at the soil surface, as the soil probe is driven or pushed into the soil a small amount of herbicide can smear from the surface into the subsurface soil zone, thus resulting in a falsepositive herbicide detection from lower soil zones. A possible procedure to reduce this contamination is to take the 0 - to $10-\mathrm{cm}$ soil sample using a larger diameter core, such as a $10-\mathrm{cm}$ golf cup cutter, and removing the surface soil sample. Now that removal of the possible area of contamination has occurred, an additional sample can then be extracted from lower subsurface zones using a sampler of smaller diameter, perhaps 2.5 to $5 \mathrm{~cm}$, that can then be inserted to the appropriate depth.

Sample Storage and Processing. Often forgotten aspects of these studies are sample storage and processing. Ideally, long-term storage should be monitored on a daily basis to verify sample integrity over time. Freezers should be checked and notations made into a logbook to verify that storage conditions are adequate. Easy-to-use and accurate long-term temperature recording equipment is available that can help automate this important task. Before sample storage, protocols and plans should be prepared to deal with any power failure or equipment malfunctions. If long-term $(>1 \mathrm{yr})$ sample storage is anticipated, the scientist should consider having fortified samples of known concentrations to be stored with soil samples for comparison when the samples are analyzed. For herbicides, storage at around $-10 \mathrm{C}$ is adequate to maintain sample integrity indefinitely, such that $-80 \mathrm{C}$ freezers are not required.

Sample processing before extraction is a potential source of error. Normally, a small subsample ( 5 to $50 \mathrm{~g}$ ) of the aggregate composite sample is used for analysis; thus, sample homogenization is essential to reduce data variability. In many environmental sample analysis projects, this sample homogenization step is the most difficult and time-consuming (Penning and Altschuck 2013). One approach is to thaw the sample and thoroughly mix by hand while the soil sample is still inside the plastic bag. This procedure is subject to variation between different technicians. Mechanical homogenization methods will require special equipment and procedures and may require the use of a refrigerant to keep the sample cold (Penning and Altschuck 2013). Care should be taken not to alter the herbicide soil concentration by excessive drying or exposure to room temperatures for extended intervals. Once the soil sample is homogenized the next step is usually extraction, although bioassays may also be used.
Chemical Concentration Determination. Usually, the determination of chemical concentrations is done under more controlled conditions than the field portion of the study. Historically, bioassays were used to determine the presence and quantity of herbicides in soils (Sunderland et al. 1991). Bioassays have several advantages, such as not requiring expensive analytical instruments, decreased use of organic solvents, and a direct measurement of biological activity of the herbicide in the soil. Some disadvantages of bioassays include lack of reproducibility, variable sensitivity due to plant growth variations, a lag time to obtain results, and, depending on the method, time-consuming procedures (washing soil off roots, drying, weighing, etc.) (Sunderland et al. 1991). Bioassays normally employ a standard curve of known herbicide concentrations that is prepared using untreated soil from the same field and fortifying to a concentration range appropriate for the application dosage. Several biological parameters may be measured using the bioassay species such as shoot fresh weight, shoot dry weight, root length, or other root parameters. The choice of the bioassay indicator species is dictated by the herbicide sensitivity of that assay, and at times bioassays can be sensitive to minute herbicide concentrations in the soil (Sunderland et al. 1991). Herbicides with high per-unit activity and low use rates, such as sulfonylureas, have been examined using soil bioassays. The quantitative nature of herbicide concentrations determined by bioassays is largely determined by the quality of the standard curve and the resultant regression analysis of that curve.

Most current researchers do not use bioassays to determine herbicide concentrations, given the tremendous improvements in mass spectrometry (MS) technology, including gas chromatography or liquid chromatography coupled with MS detectors. A typical sequence used is to:

1. weigh a designated portion of soil into the extraction vesicle, such as a bottle or vial,

2. extract with an organic solvent, possibly using solid phase extraction cleanup procedures (Lehotay et al. 2005), and

3. analyze chemically an aliquot of the extractant.

The specific chemical analysis procedure is dependent on the herbicide being examined given the various chemical attributes, such as thermal stability, chromaphore or lack thereof, intrinsic volatility of the parent and its metabolites, and other factors. The type of instrument used may also be 
dictated by the level of sensitivity needed for a given analysis; for example, a herbicide that is applied at $10 \mathrm{~g} \mathrm{ha}^{-1}$ will need a more sensitive analytical procedure than one that is applied at $\sim 1,000 \mathrm{~g} \mathrm{ha}^{-1}$.

The analytical procedure that is selected needs to fulfill the basic three tenets of a good method: accuracy, precision, and sensitivity. Accuracy is the extent to which a given measurement agrees with the standard value for that measurement. Precision is the quality that a given group of measurements are all in agreement and have small errors among them. Both accuracy and precision are important, but they reflect different attributes of the method. Sensitivity is a measure of the smallest concentration hat the analytical method can determine and is expressed as a limit of detection (LOD) and the limit of quantitation (LOQ). The LOD is the smallest concentration in which the herbicide of interest can conclusively be stated to be present in that sample, but the exact quantitative amount is somewhat uncertain. The LOQ is the smallest concentration that can be conclusively known with certainty, and that number can be used in quantitative analysis, such as regression analysis. The LOQ requires a greater signal-to-noise ratio of the instrument and is higher than the LOD. Other factors to consider in chemical analysis for herbicides include the cost of solvents (including purchase and subsequent disposal), availability and cost of various analytical instruments (which can be $>\$ 100,000$ ), the technical expertise needed to operate and maintain these complicated instruments, cost of expendable supplies, and other factors.

Another important consideration of the analytical method is the percent recovery in your particular soil-herbicide relationship. A good procedure is to fortify the soil to be examined with a known amount of herbicide and then process that fortified sample with your methodology. The percent recovery should be near $100 \%$, but acceptable values are from 80 to $120 \%$ of applied, depending on the complexity of the methods. Soils can vary substantially in their interferences, depending largely on the amount of organic matter present. Soil $\mathrm{pH}$ extremes may also impart difficulties to some analytical methods. Final herbicide concentrations should be corrected for percent recovery and for soil moisture content in the samples, especially since soil moisture will not be constant across all sampling events.

Regression Analysis. To calculate a meaningful statistic for use in simulation modeling or to discuss the data, regression analysis is essential. Historically, the first-order equation has been used to describe the dissipation of herbicides over time in field soils (Brown et al. 1996). The reader is encouraged to see the cogent paper by Aldworth and Jackson (2008) with respect to appropriate statistical tools for analysis of environmental fate data sets. In this report, we provide a broad background on the topic and provide clarity on the often biphasic nature of herbicide dissipation in surface soil studies. A potential analysis tool has also been made available through a web portal that allows for specific examination of individual data sets using this regression concept (Stone Environmental Inc. 2013).

Lab Studies. Another type of herbicide degradation study is conducted under controlled laboratory conditions in an attempt to determine the relative role of microbial compared with chemical degradation pathways for that specific herbicide. These are accomplished by taking soil with no herbicide residue and then fortifying them with a known concentration. Usually technical or analytical herbicide is dissolved in a solvent and then added to the soil. If the water solubility of the herbicide is adequate, an aqueous solution is prepared and used to fortify the soil directly. The soil should be maintained at environmentally relevant soil water content. Many herbicides have low water solubility, and thus an organic solvent is used to prepare a solution that is used to fortify the soil. An important aspect if an organic solvent is used is to add the herbicide-containing solution to the soil and then allow the organic solvent to evaporate, so as not to have a negative effect on soil microorganisms. A typical lab study involves fortifying a large number of replicate soil samples, usually contained in vials, tubes, or bottles, and then allowing them to degrade the herbicide over a time course similar to a field study. These studies are often performed in a controlled environment, such as growth chambers or incubators, and important parameters are the incubation temperature and the soil moisture content.

Another permutation on these lab studies is to include a treatment in which the soil has been sterilized. The difference between sterile (degradation due only to chemical processes) and nonsterile (degradation due to both chemical and microbial processes) indicates the relative importance of microorganisms to the decrease in herbicide concentration of that particular herbicide (Kruger 
et al. 1997). Microbial activity is often an important degradation pathway for many herbicides, and these types of studies can provide important insight into this phenomenon. The previous exposure of soil microorganisms can favor the more rapid degradation of a given herbicide, causing the phenomenon of enhanced herbicide degradation (Walker and Welch 1991). This enhanced herbicide degradation can be confirmed or at least examined using these laboratory techniques.

To conduct laboratory herbicide degradation studies properly, a few cautionary aspects should be mentioned. The soil should be collected from a representative agricultural or environmental scenario and used while still fresh. Immediate use of the soil will not allow for radical changes in the populations, species, relative growth rates, and so on of the microorganisms. A soil sample that has been dried in an oven and then stored for multiple years in a jar has greatly reduced microbial activity. Using this soil in a microbial degradation study will probably indicate much lower microbial degradation than is actually happening under field conditions. The guiding principle is to have the soil environment most closely relevant to what is actually occurring in the field, including water content, temperature, and nutrient content.

A Case History for an Example. For illustration purposes, the methods section from an example of a field dissipation study has been excerpted and printed here for consideration (Mueller and Steckel 2011). Pyroxasulfone, S-metolachlor, acetochlor, and dimethenamid were applied at $1.5 \mathrm{~kg} \mathrm{ha}^{-1}$. This was approximately equal to the standard use rates for chloroacetamides but higher than the standard rate for pyroxasulfone. This common rate was used to allow for chemical detection of each component. Each herbicide was examined as a separate treatment and was sprayed on individually replicated plots. Each herbicide was applied the same day as planting using a six-nozzle spray boom that applied chemical to the entire width of the 3-m plot. The herbicide dissipation study was maintained weed-free by applying POST glyphosate as needed.

Soil samples from 0 to $8 \mathrm{~cm}$ depth were collected using a hand-held, $10-\mathrm{cm}$-diam golf cup cutter. This sampler collected a large volume of soil and performs well under a variety of soil conditions. Two soil samples from each plot were placed into a plastic bag that was closed and immediately placed in a cooler and then quickly $(<30 \mathrm{~min})$ into a freezer. Care was taken not to allow samples inside the bags to remain in the sun, possibly degrading the herbicides. Soil samples were collected the day of application and at approximately 1, 2, 3, 4, 6, 8, and 10 wk after application.

The chemical analysis was based on previous work with a similar soil (Gallaher and Mueller 1996). For sample processing, the soil sample in each bag was allowed to thaw for approximately $30 \mathrm{~min}$ and then thoroughly homogenized, and $40 \pm 0.5 \mathrm{~g}$ of moist soil was placed into a $250-\mathrm{ml}$ low -density polyethylene Nalgene bottle. The samples in the bottles were refrozen. Herbicide concentrations were determined using methanol (liquid chromatography grade or lower) extractions of $2 \mathrm{ml}$ of solvent per gram of soil. Extractions were performed by shaking samples on a reciprocating shaker for $15 \mathrm{~h}$. When this sequence is used, the samples are put on shaker at $\sim 5$ P.M. and then removed from the shaker the next morning at $\sim 8$ A.M. It is normal for heat to be generated by the mechanical action of the shaker, so an insulating layer may be placed on the bottom of the shaker assembly to prevent high temperatures for those samples at the bottom of the shaker. When the 250$\mathrm{ml}$ bottles are removed from the shaker and allowed to equilibrate statically, the methanol layer normally separates out in $\sim 30 \mathrm{~min}$., depending on the soil texture, with clays settling slower. If needed, samples can be centrifuged to allow for particlefree extracts before filtration. If the samples will not clear even after centrifugation, the use of a prefilter on the filtration step may be needed. Extracts were passed through $0.45-\mu \mathrm{m}$ filters (Fisherbrand $25 \mathrm{~mm}$, $0.45-\mu \mathrm{m}$ PTFE [polytetrafluoroethylene], nonsterile cat. no. P9-730-21, fishersci.com) before liquid chromatography. The separation was accomplished using a C18 column, $150 \mathrm{~mm}$ by $4.6 \mathrm{~mm}$ with 3$\mu \mathrm{m}$ packing (Phenomenex Luna column, part no. 00F4251-E0, phenomenex.com). Column temperature was $25 \mathrm{C}$. The general results were that half-lives of the examined herbicides were usually less than $20 \mathrm{~d}$, although in a dry year pyroxasulfone persisted with a half-life greater than $70 \mathrm{~d}$ (Mueller and Steckel 2011).

\section{Parting Comments}

Weed control from circa 1960 until 1996 was largely based on the residual activity of herbicides in soil applied immediately after crop planting. Many foundational herbicide/crop use patterns existed, such as atrazine in corn (Zea mays L.), fluometuron 
in cotton (Gossypium hirsutum L.) or a variety of PRE herbicides in soybean [Glycine max (L.) Merr.]. The rapid adoption of GR crops shifted many production systems away from using residual soilapplied herbicides. Given the widespread development of GR weeds, there is now renewed interest in soil-applied herbicides, at least in part because they target the GR weeds at the most susceptible time in their life cycle. Given the greater complexity in weed management systems in major row crops, herbicide behavior in the surface soil environment will remain or become a major research interest (Figure 1a).

The perspectives of scientists who work in either the field or the laboratory differ with respect to herbicide dissipation studies. The field scientist works at large scales, perhaps hectares in size; has constantly changing experimental conditions; endures variable weather and a constantly changing crop; and manages the crop for nonherbicidal factors, such as insect or plant disease control. Contrast this high entropy state of the field researcher to the laboratory technician: in the laboratory the temperature is constant, with precise weighing of the same amount of soil for each sample, instrumentation that is carefully calibrated each day, and so forth. In our experiences, we have observed a difference in perspective between the field scientists and the lab chemists that eventually analyze the samples generated by the field scientist. The constraints and concerns of the two groups are different. Nevertheless, we hope this methods paper will provide insight to both the field and laboratory researcher's perspectives.

\section{Acknowledgments}

Helpful comments on this paper provided by three anonymous reviewers and Rachel Fulton are appreciated.

\section{Literature Cited}

Aldworth J, Jackson SH (2008) Statistical tools for determining appropriate selection of regression models for analysis of environmental fate datasets. Pest Manag Sci 64:536-543

Arthur EL, Barefoot AC, Clay VE (2003) Terrestrial Field Dissipation Studies: Purpose, Design, and Interpretation. ACS
Symposium Series 842. Washington, DC: American Chemical Society, $343 \mathrm{p}$

Blumhorst MR, Mueller TC (1997) Quality control procedures in herbicide field dissipation studies. Weed Technol 11:832-837

Brown BA, Hayes RM, Tyler DD, Mueller TC (1996) Effect of long-term cover crop and tillage system on fluometuron dissipation from surface soil. Weed Sci 44:171-175

Corbin M, Eckel W, Ruhman M, Spatz D, Thurman N, Gangaraju R, Kuchnicki T, Mathew R, Nicholson I (2006) NAFTA Guidance Document for Conducting Terrestrial Field Dissipation Studies. http://www.epa.gov/oppefed1/ecorisk ders/terrestrial_field_dissipation.htm. Accessed March 5, 2014

Gallaher K, Mueller TC (1996) Effect of crop presence on persistence of atrazine, metribuzin, and clomazone in surface soil. Weed Sci 44:698-703

Kruger EL, Rice PJ, Anhalt JC, Anderson TA, Coats JR (1997) Comparison fates of atrazine and deethylatrazine in sterile and nonsterile soils. J Environ Qual 26:95-101

Lehotay SJ, de Kok A, Hiemstra M, van Bodegraven P (2005) Validation of a fast and easy method for the determination of residues from 229 pesticides in fruits and vegetables using gas and liquid chromatography and mass spectrometric detection. J AOAC Int 88:595-614

Leng ML, Leovey EM, Zubkoff PL (1995) Agrochemical environmental fate: state of the art. Boca Raton, FL: Lewis Publishers. $410 \mathrm{p}$

Locke MA, Bryson CT (1997) Herbicide-soil interactions in reduced tillage and plant residue management systems. Weed Sci 45:307-320

Mueller TC, Steckel LE (2011) Efficacy and dissipation of pyroxasulfone and three chloroacetamides in a Tennessee field soil. Weed Sci 59:574-579

Penning H, Altschuck A (2013) Optimization of sample homogenization for a pesticide residue analysis: soil-an example for a difficult matrix. Picogram Volume 84, Page 127, Abstract 156 in Proceedings of the American Chemical Society. Indianapolis, IN: American Chemical Society

Phelps W, Winton K, Effland WR (2002) Pesticide environmental fate: bridging the gap between laboratory and field studies. ACS Symposium Series 813. Washington, DC: American Chemical Society. $236 \mathrm{p}$

Sarmahm AK, Sabadie J (2002) Hydrolysis of sulfonylurea herbicides in soils and aqueous solutions: a Review. J Agric Food Chem 50:6253-6265

Stone Environmental Inc. (2013) Agrochemical Fate and Exposure Resources. Downloads. http://www.stone-env.com/ agchem/agres.php\#agdownload. Accessed September 23, 2013

Sunderland SL, Santelmann PW, Baughman TA (1991) A rapid, sensitive soil bioassay for sulfonylurea herbicides. Weed Sci 39:296-298

Walker A, Welch SJ (1991) Enhanced degradation of some soilapplied herbicides. Weed Res 31:49-57

Received October 24, 2013, and approved March 20, 2014. 